\title{
Framework Surgery for Treatment of Unilateral Vocal Fold Paralysis
}

\author{
James J. Daniero • C. Gaelyn Garrett • \\ David O. Francis
}

Published online: 27 February 2014

(c) Springer Science+Business Media New York 2014

\begin{abstract}
Laryngeal framework surgery is the current gold standard treatment for unilateral vocal fold paralysis (UVFP). It provides a permanent solution to glottic insufficiency caused by injury to the recurrent laryngeal nerve. Various modifications to the original Isshiki type I laryngoplasty procedure have been described to improve voice and swallowing outcomes. The success of this procedure is highly dependent on the experience of the surgeon as it epitomizes the intersection of art and science in the field. The following article reviews the evidence, controversies, and complications related to laryngoplasty for UVFP. It also provides a detailed analysis of how and when arytenoid-positioning procedures should be considered and summarizes the literature on postoperative outcomes.
\end{abstract}

Keywords Unilateral vocal fold paralysis .

Framework surgery · Laryngoplasty ·

Arytenoid adduction - Vocal fold medialization

J. J. Daniero · C. G. Garrett - D. O. Francis

Vanderbilt Voice Center, Department of Otolaryngology, Bill

Wilkerson Center, Vanderbilt University Medical Center,

Medical Center East, South Tower, 1215 21st Avenue South,

Nashville, TN 37232-8732, USA

D. O. Francis $(\bowtie)$

Center for Surgical Quality \& Outcomes Research, Institute for

Medicine and Public Health, Vanderbilt University Medical

Center, 12th Floor, 2525 West End Avenue, Nashville,

TN 37232-8732, USA

e-mail: david.o.francis@vanderbilt.edu

\section{Introduction}

The role of the larynx in voice production is to efficiently convert aerodynamic energy from the chest and lungs into acoustic energy. Critical in this process is vocal fold adduction, which potentiates glottal valve closure. A closed valve resists subglottic pressure until the subglottic pressure threshold is exceeded, at which point the vocal folds open allowing transglottic airflow. The Bernoulli effect then entrains a mucosal wave over their surfaces, generating acoustic energy that serves as the sound source for voice. The remainder of the vocal tract acts as a resonating chamber to modulate sound into the speaking and singing voice.

The recurrent laryngeal nerve innervates all intrinsic muscles of the larynx. Its fascicles are contained within paired vagus nerves, which originate in the nucleus ambiguus located in lateral portion of the rostral medulla. These give rise to branchial efferent motor fibers that travel caudally within the carotid sheath into the chest where the recurrent laryngeal nerves branch off the main vagal trunks, wrapping around the aortic arch and subclavian artery on the left and right side, respectively. They then travel cranially within the tracheoesophageal groove to enter the larynx with the inferior laryngeal artery and vein near the cricoarytenoid joint. Thereafter, the nerves distally arborize to innervate ipsilateral intrinsic laryngeal muscles in a 4:1 ratio between the adductor and abductor compartments [1, 2]. It should be noted that this ratio is based on animal studies; the exact proportion in humans has not been fully characterized.

Vocal fold motion results primarily from the actions of the intrinsic laryngeal muscles on the cricoarytenoid joint. Internal or external rotation of the arytenoid cartilage causes the ispilateral vocal fold to adduct and abduct, 
respectively. However, the arytenoid is a complex threedimensional joint that rotates in both the medial-lateral plane and also providing forward and backward tilt [3]. Thus, adduction is more accurately accomplished by the medial-inferior rotation and tilting of the arytenoid cartilage through the contraction of thyroarytenoid, lateral cricoarytenoid, and interarytenoid muscles.

\section{Epidemiology of Unilateral Vocal Fold Paralysis}

Unilateral vocal fold paralysis (UVFP) has major health and quality of life implications. It is caused by injury to the recurrent laryngeal nerve and is iatrogenic in approximately half of cases, most commonly from thyroidectomy or anterior cervical disc fusion surgeries (ACDF) [4-7]. Increased indications for head, neck, cervical spine, and cardiothoracic surgical procedures have resulted in a rapidly growing population at risk for UVFP. Specifically, UVFP complicates up to $15 \%$ [8-10] and $11 \%[11,12]$ of thyroidectomies and ACDF procedures, respectively; in recent decades, rates of these procedures have increased three- and eightfold [13-15], with an expected collinear rise in UVFP incidence. This combined with other etiologies (e.g., traumatic, idiopathic, malignant) puts an increased proportion of patients at risk for this condition.

The health and quality of life ramifications are significant. Studies of non-UVFP-attributable voice disorders report health-related quality of life (HRQOL) implications and work productivity losses comparable to patients with asthma, acute coronary syndrome, and depression, with 1:10 affected individuals filing short-term disability claims [16•]. Patients with UVFP-attributable voice disorders have substantially worse HRQOL $[17,18]$ and even more productivity losses. From a health perspective, $60 \%$ of patients suffer from dysphagia [19] (23\% with aspiration) [20] and $75 \%$ new onset dyspnea [19].

Variability exists in the approach to surgical management. The degree of spontaneous recovery and thus timing of intervention vary based on the severity and permanency of neurological injury [21, 22•]. The basic tenet of treatment is to recapitulate the laryngeal valve, thereby correcting glottic insufficiency. Although patients benefit significantly from interventions [23•, 24], some debate exists as to optimal treatment type(s) and their timing(s) (e.g., injection augmentation, reinnervation, laryngoplasty, and arytenoid repositioning). This likely derives from a paucity of rigorous comparative effectiveness studies that directly compare different treatment algorithms and interventions [23•]. It is important to recognize that management is necessarily based on symptom severity; therefore, its quantification and patient participation in decision-making are critical.

\section{History of Framework Surgery}

The ideal procedure to address glottal insufficiency would be to recreate the natural motion of the cricoarytenoid joint and tensing of the thyroarytenoid muscles to reconstitute physiologic glottis closure, reverse dysphagia, and improve voice quality, while maintaining adequate airway for respiration. This goal is yet to be realized. Currently, the most efficacious treatment is medialization of the impaired vocal fold with injectable fillers (temporary) or manipulation of the laryngeal framework (permanent). Erwin Payr first described laryngeal framework surgery for vocal fold medialization in 1915 [25]. However, in the pre-antibiotic and pre-steroid era, it was associated with significant risk of infection and airway obstruction, and it was therefore largely abandoned. Isshiki et al. [26] reintroduced the concept in 1974, describing four types of framework or laryngoplasty surgeries: type I: compression (medialization); type II: expansion (lateralization); type III: shortening (relaxing); type IV: lengthening (tensing) [2]. Type I laryngoplasty has become the preferred treatment for UVFP. In fact, a 2008 survey of board-certified otolaryngologists found that type I laryngoplasty accounted for $85 \%$ of all framework surgery performed in the US [27].

It was recognized early that medialization alone does not always restore adequate voice in settings of vocal fold height mismatch or a persistent posterior glottic gap. Arytenoid adduction, first described in 1978, was devised to address these issues [28]. In essence, it involves a suture placed through the muscular process and secured anteriorly in the apex of the thyroid cartilage to simulate contraction of the lateral cricoarytenoid muscle, thus controlling the position of the vocal process in both a vertical and horizontal plane. Isshiki et al. [28] demonstrated this to effectively close the persistent posterior glottic gap. Koufman [29] and Netterville [30] popularized these procedures in the US. Woo et al. [10] reinforced its role in framework surgery in a report on 20 patients with poor results after laryngoplasty for UVFP. Twelve were found to have a persistent posterior glottic gap, and all had voice restored with the addition of arytenoid adduction during revision surgery.

\section{Pre-operative Considerations}

Evaluation and management of UVFP involve detailed history, pre-operative laryngoscopy, pre-surgical counseling, and speech language pathologist (SLP) evaluation. Other tests variably employed include laryngeal electromyography (LEMG), swallow assessment (e.g., modified barium swallow study), acoustic and aerodynamic testing, and pulmonary function testing. Their roles are briefly discussed below. 
History

A complete history is critical in the pre-operative evaluation of UVFP patients. Of particular interest are the duration and etiology of the paralysis, associated symptoms, and how the condition affects their ability to work and quality of life. Medications should be carefully reviewed and patients asked whether they are on aspirin, non-steroidal anti-inflammatory medications, anticoagulants (e.g., heparin, warfarin), or supplements with anticoagulant properties (e.g., St. John's wort). Those patients who take these medications should consult with their prescribing physician about whether they can safely come off these medications for surgery, as risks of hemorrhage and related sequelae, although rare, may be increased in this subset of patients [31].

Moreover, it is important to inquire whether any previous interventions for UVFP have been attempted. Specifically, many patients may have previously undergone temporary injection augmentations. If so, it is important to accurately determine when the procedures were performed and the durability of the injectable used. Residual injectable temporarily changes the three-dimensionality and geometry of the paraglottic space and can significantly impact the ultimate result of the type I laryngoplasty. If there is concern whether a residual injectable is present, a pre-operative CT may help confirm that the absorbable material has been completely resorbed before considering framework surgery.

\section{Laryngoscopy}

Laryngoscopy is the principal tool in the pre-operative evaluation of vocal fold mobility. Most laryngologists agree that flexible rather than rigid laryngoscopy provides the most accurate assessment of vocal fold mobility and degree of glottic insufficiency [32, 33]. The postulated reason is that patients posture or splint more during rigid laryngoscopy, thus giving the appearance of movement abnormalities that are not necessarily present on flexible examination. Videostroboscopy provides further information on associated vocal fold vibratory perturbations and also provides a more complete evaluation of vocal fold closure. For example, aperiodicity may indicate a vocal fold height mismatch. However, despite its critical role, laryngoscopy is an imperfect diagnostic test as it only provides two-dimensional information on glottic configuration.

\section{Presurgical Counseling}

Proper patient selection is crucial to optimize framework surgery results. Patients must be counseled that this surgery is most commonly performed awake and that they will be required to lay supine on the operating room table for between $45 \mathrm{~min}$ and $3 \mathrm{~h}$ depending on technique and surgeon preferences. Patients should understand that operating room positioning can cause claustrophobia and that they may have a flexible laryngoscope in place either throughout or intermittently during their operative experience. Therefore, patients with severe chronic cough, orthopnea, anxiety, or psychological instability may not tolerate this type of surgery. However, they should be reassured that every effort will be made to make them comfortable and that communicating their intraoperative feelings and sensations is important to a successful outcome.

Patient expectations should be managed through a detailed pre-operative discussion of the expected outcomes. Although $92 \%$ reported satisfaction with their voice after type I laryngoplasty, $87 \%$ of patients still considered their voice abnormal. Despite surgical treatment, $27 \%$ ultimately required employment changes as a result of a disordered voice [34]. Therefore, surgeons should inform the patient that a normal voice is often not attainable. Instead, the goal of surgery should be to significantly improve glottic efficiency and to reduce the effort needed to voice. The variable effect of laryngoplasty on reversing dysphagia should also be discussed [35-37].

Furthermore, patients should be counseled regarding the potential need for revision surgery. A national survey published in 1998 reported a revision rate of $5.4 \%$ [38]. Although commonly thought of as a reversible procedure, animal model studies suggest that there may be changes to the cricoarytenoid joint that persist after implant removal [39]. These are important considerations for the patient. In addition, other risks must also be reviewed including bleeding, infection, implant extrusion, and potential for intra- and postoperative airway obstruction. There are also some data suggesting a higher risk for airway complications with future procedures requiring an anesthetic [14]. A risk exists for postoperative dyspnea from medialization, but airflow studies, to date, have not found the procedure to significantly increase respiratory resistance [40]. In fact, it is the authors' experience that patients often report improved subjective breathing and exercise tolerance due to the ability to regulate glottic airflow and perform the Valsalva maneuver.

\section{Speech Language Pathologist Evaluation}

A multidisciplinary approach is required for management of UVFP. In particular, SLPs have an important role [4143]. A full voice evaluation and initial counseling by a SLP will help prepare the patient for surgery and raise awareness of the relationship between breath support and voicing [44]. This may facilitate unloading of compensatory supraglottic hyperfunction during the actual procedure and 
optimize outcomes. Moreover, patient satisfaction and compliance may be improved with pre- and postoperative voice therapy. Some surgeons even request the assistance of the SLP intraoperatively for help with perceptual analysis, to facilitating unloading, and to suggest appropriate voicing tasks, while the surgeon focuses on the adjustments required. Although insufficiency should largely be resolved with framework surgery, voice therapy can help mitigate inadequate breath support that can impair the postoperatively recovery process and confound functional voice outcomes.

\section{Surgical Timing}

In general, type I laryngoplasty is a permanent solution and therefore should only be considered in patients who are refractory to conservative measures (i.e., speech therapy) and expected to remain persistently symptomatic from UVFP without treatment. Most practitioners accept that a waiting time between 6 and 12 months is appropriate before considering framework surgery [21]. This time period is based largely on dogma; however, there are some data from peripheral nerve studies indicating that the rate of axonal regeneration is between 0.5 and $1.5 \mathrm{~mm} /$ day [45-48]. In cadaver studies, the left and right recurrent laryngeal nerves measured 136 and $75 \mathrm{~mm}$ in length, respectively [49]. Therefore, a period of $\sim 272$ (left) and 150 days (right) would be necessary for recurrent laryngeal nerves to reach the laryngeal end organ if at least four assumptions are met: (1) axontomesis or nerve injury occurred near its origin, (2) the nerve length is relatively constant in the population, (3) regeneration rate is fixed, and (4) it occurs at $0.5 \mathrm{~mm} /$ day. Obviously, these are gross suppositions, and axonal regeneration does not equate to either return of movement or synkinesis. Illustrating the uncertainty in these estimates are case reports finding delayed recovery 18 months to 4 years following symptom onset [50, 51]. Furthermore, the pathophysiology is often deemed idiopathic and degree of injury rarely quantifiable. Nonetheless, this logic is the likely basis for current practice.

There are circumstances when early laryngoplasty may be indicated. First, if the recurrent laryngeal nerve was either volitionally or unintentionally sectioned, the likelihood of developing sufficient synkinesis or movement is low $[30,52]$. However, caution must be exercised when considering early and immediate type I laryngoplasty even in the setting of recurrent laryngeal nerve sectioning. Studies of peripheral nerve sacrifice indicate that denervation atrophy begins as early as 2 weeks, and $\sim 75 \%$ of muscle mass is lost by 4 months $[53,54]$. Thus, a several month waiting period is suggested before permanent medialization to allow for muscle atrophy to occur in order to prevent an under-medialized result. A second situation may arise when LEMG is performed and demonstrates signs of poor prognosis. Controversy remains concerning whether LEMG findings alone are sufficient to warrant early intervention. To address this, Rickert et al. [22•] pooled data from literature on LEMG as a prognostic tool for recovery from UVFP. They reported a $90.9 \%$ probability that a person will not recovery motion if they demonstrate evidence of "high grade injury" on EMG, but that EMG was an indifferent predictor of recovery (55.6\%). It was therefore argued that patients demonstrating presence of spontaneous activity [55-59] or absence or reduced motor unit potentials $[56,57,60-62]$ at 6 months may be good candidates for early intervention [22•].

There are significant methodologic limitations to this study. First, the meta-analysis did not provide information regarding full search results and yield. Further, there was no mention of double review/abstraction, and they did not assess the rigor of studies (i.e., evidence level, methods). Moreover, not all studies included used the same prognostic criteria; therefore, results cannot be appropriately pooled. Second, LEMG timing differed in studies, which necessarily impacts the degree of reinnervation and EMG findings. Third, there was no mitigation of patient selection bias. Methodological issues surrounding data heterogeneity limit the ability to pool data into a meta-analysis. Metaanalytic statistics can adjust for variable study quality and heterogeneity using quality effects and random effects modeling, respectively. Finally, a fixed effect model should also have been considered to avoid results heavily biasing toward larger studies while minimizing those from smaller studies. Thus, rigorous meta-analytic statistical assessment would have provided more accurate conclusions.

Nonetheless, their review is an important first attempt, but its results must be interpreted carefully considering the above methodological limitations and that, assuming the accuracy of findings, one out of ten patients might undergo unnecessary surgery using these criteria. As surgeons, we want to offer a definitive and expeditious treatment to reduce the associated symptom and quality of life burden by offering early intervention. Injection augmentation offers this opportunity and can temporize patients for the appropriate waiting period to determine if movement will be restored or adequate synkinesis developed to reverse glottic insufficiency. This approach obviates the risk of premature, potentially unnecessary definitive surgical intervention. LEMG is a useful adjunct, but it remains a qualitative tool whose role in directing management of UVFP needs further definition.

\section{Other Pre-operative Testing}

Patients with UVFP have disrupted airway protection and/ or sensory deficits and are thus prone to aspiration [63-65]. 
Therefore, pre-operative evaluation should inquire into whether affected patients have had pneumonia and/or have noticed increased liquid or solid food dysphagia since symptom onset [66, 67]. In these situations, swallow assessment (e.g., modified barium swallow study) is helpful to characterize the degree of penetration, aspiration, any abnormalities at the cricopharyngeus, and to teach and reinforce safe swallowing maneuvers [65]. Aeroacoustic and pulmonary function studies are used by some to measure glottic airflow rates and glottic resistance, but are considered supplemental by most surgeons.

\section{Issues Related to High-Vagal Injury}

Special considerations in high-vagal paralysis involve complex swallowing dysfunction beyond issues related to glottic insufficiency. Dysphagia after vagal paralysis is multifactorial in nature due to the additional loss of sensation via the internal branch of the superior laryngeal nerve and paralysis of the vagal contribution to the pharyngeal plexus. This results in dysregulation of the pressure wave required to propel the bolus past a larynx that has lost protective mechanisms. To complicate matters further, patients may also suffer from incomplete cricopharyngeal relaxation due to loss of vagal input $[68,69]$. Therefore, pre- and postoperative modified barium swallow studies should be considered to assess for severity of aspiration and levels of injury. Assessment for hypernasal speech, nasal regurgitation, pooling of secretions, and velopharyngeal insufficiency by flexible endoscopy should be included in the pre-operative evaluation of these patients. Adjunctive procedures such as pharyngeal flap, arytenoid adduction, and cricopharyngeal myotomy may also be required $[66,70,71]$. Extensive swallowing therapy is also often required in these patients both pre- and postoperatively [72]. Moreover, patients presenting after anterior cervical disk fusion procedures may complain of solid food dysphagia, presumed to be caused by pharyngeal plexus injury [73, 74]. This condition may not improve with intervention, and therefore affected patients must be counseled regarding realistic expectations [75].

\section{Perioperative Considerations}

Perioperative considerations for laryngeal framework surgery are unique since patient interaction is required to optimize outcomes. Some debate exists over the amount of sedation given. The procedure can be successfully performed with only local anesthesia. For example, the authors' institution typically uses equal parts bupivacaine and lidocaine. However, various methods of sedation can be administered, including remifentanil, dexmedetomidine, propofol boluses, and intermittent administration of benzodiazepines. A balance must be struck between patient comfort and their ability to fully cooperate during the procedure. A short-acting medication can provide analgesia and/or amnesia during the surgical approach and be titrated off prior to the critical voicing portion of the case. However, efforts should be made to prevent patient disinhibition, movement during the procedure, and apnea from respiratory depression or upper airway collapse. In addition, the level of sedation may interfere with the ability to achieve best effort phonation, thus limiting the ability to optimize the framework surgery outcome. Significant variability in sedation exists based on surgeon preference. Regardless of sedation plan, excellent communication between the surgeon and the anesthesiologist is required to assure the ideal result, while keeping the patient comfort level and satisfaction high.

Another consideration in the operative setup for laryngoplasty is the requirement for two separate fields to correlate endoscopic confirmation of medialization and voice improvement. This technique is particularly helpful to confirm proper implant positioning and prevent over-medialization of the anterior vocal fold. In order to keep the operative field for the implant sterile, various techniques have been developed to allow access to both fields without contamination. As previously described by Wanamaker et al. [76], our institution utilizes a sterile plastic drape suspended to an IV pole that wraps around a flexible videolaryngoscope. The scope passed through the nose is secured in the optimal position above the larynx and suspended from the IV pole. This allows an assistant to operate and adjust the laryngoscope sterilely through the drape while the surgeon manipulates the paraglottic space during voicing tasks. Others have described only placing the laryngoscope intermittently during key portions of the case and checking for vocal fold position. This method avoids the need for the patient to have an endoscope placed in their nose for the duration of the case. However, the surgeon or their assistant must break sterility to operate the scope.

Patient positioning is an important concern when performing surgery under local anesthesia, particularly when it has the potential to last several hours. A beach chair position with a pillow underneath the knees takes strain off of the lower back and neck. Also, a neck roll with or without a shoulder roll is often used to support the neck when held in an extended position for surgery. Ideally, total operative time should be minimized to $<3 \mathrm{~h}$ (if possible) because of patient intolerance.

\section{Surgical Approach}

Laryngeal framework surgery for UVFP typically involves indirect medialization of the vibratory margin of the vocal 
fold by augmenting the paraglottic space with permanent implant material. Steps in the process include: (1) creation of the laryngoplasty window; (2) implant material choice, sizing, and placement; (3) considerations for arytenoid adduction. Each will be discussed below.

\section{Laryngoplasty Window}

A window in the thyroid cartilage is created immediately lateral and as close to the level of the affected vocal fold as possible, which allows access to the paraglottic space. A number of approaches are employed. Some advocate the use of a drill to create "sounding" holes in the thyroid cartilage that can be used to localize the position of the vocal fold using lacrimal probes. Whether or not this is performed, a window must then be created. In situations of ossification along the inferior aspect of the thyroid cartilage, removal of dense cartilage for the window can be accomplished with a small oscillating saw or, more commonly, a drill and small round burr (e.g., 3-mm-round cutting burr). Occasionally, younger patients have soft cartilage and the window can be fashioned using a scalpel. A small bone curette is often employed to sharpen corners and edges of the window.

The window size and shape vary based on the choice of implant material and surgeon preference. For Silastic ${ }^{\circledR}$ implants, placement of the anterior border of the window is typically $5 \mathrm{~mm}$ from midline in female patients and $7 \mathrm{~mm}$ from midline in male patients because of the more obtuse anterior angle of the thyroid cartilage in women. This prevents over-medialization of the anterior vocal fold and resultant pressed or strained voice quality. Moreover, the window should be placed $\sim 3 \mathrm{~mm}$ from and parallel to the inferior border of the thyroid cartilage. The Gore-Tex ${ }^{\circledR}$ laryngoplasty window is created approximately $1 \mathrm{~cm}$ posterior to midline. This smaller $4-10-\mathrm{mm}$ window may be placed a few millimeters anteriorly or posteriorly to customize placement of implant material as needed [77].

There is variation in the approach to managing the inner perichondrium of the thyroid cartilage. Some surgeons leave it intact, preferring to elevate the inner perichondrium as an intact layer, thereby providing a platform on which they can medialize the vocal fold along a broad front, theoretically reducing the risk of implant extrusion [78]. However, others argue that incising and removing the inner perichondrium allow for more precise contoured medialization without increasing the risk of implant extrusion [76]. In this approach, a plane is created medial to the inner perichondrium superiorly, inferiorly, and posteriorly to create adequate space for implant placement. Regardless, the ultimate goal in medialization laryngoplasty is to improve glottic competence and provide an optimal voice outcome. Thus, effort is made to medialize the lower lip to improve vocal fold contact while limiting the strain observed with supraglottic medialization. Medialization of the arytenoid with complex posterior extension implant remains controversial. Since first described by Isshiki [79], some surgeons have found this approach moves the arytenoid into a less advantageous position than is possible with direct arytenoid repositioning [80]. However, the authors find that customization of the laryngoplasty implant can allow an anterior approach to arytenoid repositioning without requiring arytenoid exposure and formal suture adduction.

\section{Implant Material, Sizing, and Placement}

Implant materials are divided into autologous and synthetic. Autologous implant materials include fat, fascia, muscle, and cartilage. Although these materials have been reported to be effective, they are not widely used because of the unpredictable nature of their long-term medialization related to variable absorption and migration. Synthetic materials include Silastic ${ }^{\circledR}$, Gore-Tex ${ }^{\circledR}$, and others (Table 1). Freeform (e.g., Phonoform ${ }^{\circledR}$ ) and pre-sized (Montgomery ${ }^{\circledR}$ ) implants require patient-level customization by the surgeon either by intraoperative manipulation or choosing the appropriate size. Freeform Silastic ${ }^{\circledR}$ is hand carved with a scalpel to match measured medialization based on probing the paraglottic space with a depth gauge during patient voicing tasks. The point of maximal displacement and depth of medialization are used as guides to fashion a wedge implant that locks into the laryngoplasty window without the need for suture fixation. This technique offers the ability to precisely individualize medialization based on exact measurements required to achieve glottal competence. The Gore$\mathrm{Tex}^{\circledR}$ technique involves advancing a ribbon of material into the paraglottic space and titrating placement to effect based upon real-time voicing and laryngoscopy. This technique offers the ability to customize the final medialization and eliminates the time needed to carve a custom implant. If a small window is created for the Gore-Tex ${ }^{\circledR}$, no additional closure is required; however, larger windows require closure to prevent extrusion. This is commonly done with perichondrium, suture, or strap muscle. Finally, recent studies from Hoffman et al. [81, 82] have reported on a synthetic freeform wedge-shaped adjustable balloon that can be placed in the paraglottic space and sized appropriately by inflating with saline solution. Other preformed implants that are variably customizable are shown in Table 1 .

A recent survey of all board-certified otolaryngologists in the US analyzed trends in implant use by comparing practice patterns in 1998-2010 [27]. They concluded that there was a trend away from the use of Silastic ${ }^{\circledR}$ toward greater use of Gore-Tex ${ }^{\circledR}$. However, the results are skewed by incomplete categorization of implant types. Customizable Silastic ${ }^{\circledR}$ is used not only in freeform blocks, but also constitutes both 
Table 1 Summary of implant materials

\begin{tabular}{|c|c|c|c|c|c|}
\hline $\begin{array}{l}\text { Implant } \\
\text { category }\end{array}$ & Type & Material & Sizing & Company & Medialization plane \\
\hline \multirow{3}{*}{$\begin{array}{l}\text { Autologous } \\
\text { freeform }\end{array}$} & Fat & Biologic & Custom & NA & Vertical and horizontal \\
\hline & Fascia & Biologic & Custom & NA & Vertical and horizontal \\
\hline & Cartilage & Biologic & Custom & NA & Vertical and horizontal \\
\hline \multirow[t]{2}{*}{$\begin{array}{l}\text { Synthetic } \\
\text { freeform }\end{array}$} & Silastic $^{\circledR}$ & Polymerized silicone & $\begin{array}{l}\text { Custom } \\
\text { carved }\end{array}$ & Several & Vertical and horizontal \\
\hline & Gore-Tex ${ }^{\circledR}$ & $\begin{array}{l}\text { Expanded } \\
\text { polytetrafluoroethylene }\end{array}$ & Custom & Several & Vertical and horizontal \\
\hline \multirow[t]{5}{*}{$\begin{array}{l}\text { Synthetic } \\
\text { prefabricated }\end{array}$} & $\begin{array}{l}\text { Netterville } \\
\text { Phonoform }^{\circledR}\end{array}$ & Polymerized silicone & $\begin{array}{l}\text { Custom } \\
\text { carved }\end{array}$ & $\begin{array}{l}\text { Medtronic, Minneapolis, } \\
\text { MN, USA }\end{array}$ & Vertical and horizontal \\
\hline & Montgomery ${ }^{\circledR}$ & Polymerized silicone & $\begin{array}{l}5 \text { Male sizes } \\
5 \text { Female } \\
\text { sizes } \\
\text { customizable }\end{array}$ & $\begin{array}{l}\text { Boston Medical Products, } \\
\text { Westborough, MA, USA }\end{array}$ & Horizontal only \\
\hline & $\operatorname{VoCoM}^{\circledR}$ & Hydroxyapatite & 5 Sizes & $\begin{array}{l}\text { Gyrus ENT, Bartlett, TN, } \\
\text { USA }\end{array}$ & Vertical and horizontal \\
\hline & $\begin{array}{l}\text { Titanium vocal fold } \\
\text { medialization } \\
\left(\text { TVFMI }^{\mathrm{TM}}\right)\end{array}$ & Titanium & $\begin{array}{l}1 \text { Male, } \\
1 \text { Female } \\
\text { adjustable }\end{array}$ & $\begin{array}{l}\text { Heinz Kurz GmbH, } \\
\text { Dusslingen, GR, Germany }\end{array}$ & Horizontal only \\
\hline & $\begin{array}{l}\text { Adjustable balloon } \\
\text { implant }\end{array}$ & $\begin{array}{l}\text { Saline-filled silicone } \\
\text { balloon }\end{array}$ & $\begin{array}{l}1 \text { Size } \\
\text { adjustable }\end{array}$ & $\begin{array}{l}\text { Hood Laboratories, } \\
\text { Pembrooke, MA, USA }\end{array}$ & Horizontal only \\
\hline
\end{tabular}

Phonoform ${ }^{\circledR}$ and Montgomery ${ }^{\circledR}$ implants. Reanalysis of the data does show a move away from unshaped Silastic ${ }^{\circledR}$ blocks toward the use of freeform (Phonoform ${ }^{\circledR}$ ) and pre-sized (Montgomery ${ }^{\circledR}$ ) implants. In fact, Silastic ${ }^{\circledR}$ remains the most commonly used material for medialization laryngoplasty: $\sim 63 \%$ Silastic $^{\circledR}, 30 \%$ Gore-Tex $^{\circledR}$, and $7 \%$ VoCoM $^{\circledR}$. Trends toward use of preformed and pre-sized Silastic ${ }^{\circledR}$ are likely due to the reduced time required to customize the implant and reduced need for specialized training in handcarving the implant.

\section{Arytenoid Repositioning Procedures}

Medialization of the vocal fold can also be accomplished through arytenoid repositioning procedures. Isshiki et al. [28] first described this procedure in 1978 to combat persistent posterior glottic gap and vocal fold height mismatch. Today, these techniques are more often used in selected patients as an adjunct to medialization laryngoplasty in order to optimize correction of glottic insufficiency. Caution should be practiced with this approach, as too much tension on the suture will result in excessive rotation and anterior displacement of the arytenoid. Arytenoid malpositioning is manifest by the vocal process crossing the midline with bowing and shortening of the ipsilateral vocal fold [83, 84•].

In most cases, the approach to the muscular process of arytenoid is accomplished through the same operative exposure used for implant placement. Further dissection is needed posterior-laterally to exposure the posterior edge of the thyroid ala. Exposure is significantly improved by removal of the posterior portion of the thyroid ala with Kerrison rongeurs to allow easier identification of the muscular process of the arytenoid while limiting soft tissue dissection in the pyriform sinus region [85]. Better exposure limits airway edema and risk of mucosal perforation. Access to the posterior larynx can be particularly difficult in males; therefore, division of the strap muscles may occasionally be necessary to gain access to perform arytenoid surgery. Arytenoid exposure can be challenging in some patients. Anatomical studies have therefore been performed to facilitate identification. A cadaveric study by Mitchell et al. [86] found that the muscular process was located along a line extending posteriorly from the inferior border of a standard laryngoplasty window. In males, the average distance was $27 \mathrm{~mm}$, whereas in females the distance was $19 \mathrm{~mm}$. The muscular process was consistently found an average of $2.7 \mathrm{~mm}$ inferior to the midpoint between the roots of the superior and inferior cornu. Similarly, in another cadaver study by Armin et al. [87], the muscular process of the arytenoid was found halfway between the roots of the superior and inferior cornu.

Zeitels et al. [88] described an alternative method for arytenoid repositioning in 1998. This procedure involves opening the cricoarytenoid joint and placing a suture through the posteromedial cricoid facet and then 
horizontally through the body of the arytenoid or muscular process in the desired position for adduction of the vocal fold. This procedure directly places the arytenoid in the desired position and may avoid constraints of manipulating intact laryngeal biomechanics. Cricothyroid subluxation is an additional adjunctive procedure supplementing medialization laryngoplasty to add tension to the paralyzed vocal fold [89]. This procedure involves placing a suture around the inferior cornu of the thyroid lamina and securing it to the cricoid cartilage. This mimics the action of the cricothyroid muscle in placing tension on the vocal fold. In 2008, McNamar et al. [90] compared arytenoid adduction and adduction arytenopexy in an ex vivo model using the same larynx for both procedures. No statistically significant difference was demonstrated in phonation threshold pressure (PTP), glottal resistance, output-cost, or symmetry of vocal fold vibration on high-speed video. However, two separate dominant frequencies were noted after arytenoid adduction, whereas a single fundamental frequency was observed with adduction arytenopexy.

The decision of when to perform an arytenoid repositioning procedure is met with great controversy. There is a range of opinions from never to always performed, with most surgeons falling somewhere in between on the spectrum [91]. Isshiki originally developed the procedure to address persistent posterior glottic gap and vocal fold height mismatch. Subsequent studies have reinforced these indications, particularly in the setting of high-vagal paralysis and a far lateralized vocal fold [66, 92]. Others have informally suggested its use in patients that need loud projection for their occupation such as military service personnel, police persons, firefighters, and coaches. Nonetheless, it is difficult to pre-operatively predict the necessity for arytenoid adduction. $\mathrm{Li}$ et al. [93•] found no difference in blinded review of postoperative video between patients who underwent laryngoplasty either alone or in combination with arytenoid adduction. Interestingly, pre-operative videolaryngoscopy showed wider glottic gaps and great vertical height differences in those who went on to get arytenoid adduction compared to those getting medialization alone. No difference in postoperative appearance or outcome should be interpreted to mean that arytenoid adduction was necessary in those patients with wider gaps and greater height difference to achieve equivalent results to those only requiring medialization alone.

Endoscopic arytenoid adduction is a new technique that has been described [94]. This is performed under general anesthesia in order to obtain endolaryngeal exposure. Two needles are placed through the cricothyroid membrane, one penetrating needle and a loop needle. Each needle carries a suture. One is wrapped around the muscular process of the arytenoid, and the other serves to retrieve the end when hooked endoscopically. The patient is then awakened from anesthesia, and the arytenoid adduction suture is tightened appropriately to optimal voice. This technique demonstrated statistically significant improvement in maximum phonation time, mean flow rate, jitter, and shimmer over pre-operative assessment in ten patients.

Hoffman et al. [95] performed an analysis of high-speed video, acoustic, and aerodynamic measures between injection laryngoplasty and type I laryngoplasty with and without arytenoid adduction in the ex vivo canine larynx. Although this study has limited clinical application due to differences in species and the ex vivo nature of the experiment, excellent control was gained in the ability to compare acoustic and aerodynamic measures between type I laryngoplasty alone and with arytenoid adduction performed in the same larynx. This study would be difficult to perform in vivo, but does provide excellent insight into the true biomechanics of laryngeal framework surgery. Type I with arytenoid adduction provided the greatest improvement in phonatory measurements, including phonation threshold flow, phonation threshold power, and signal to noise ratio.

Chester and Stewart [91] performed an evidenced-based review of arytenoid adduction in 2003. Their review showed no clear additional benefit to arytenoid adduction when combined with laryngoplasty. Their results are actually an endorsement for the effectiveness of arytenoid adduction. Medialization with or without arytenoid repositioning shares a common goal to achieve optimal voice outcome. Mortensen et al. [96] showed that the patient population requiring arytenoid adduction is often more dysphonic pre-operatively. Arytenoid adduction is generally added to medialization when the implant alone does not achieve the desired outcome. Thus, the evidence-based finding of equivalence whether or not arytenoid adduction was added to laryngoplasty shows its usefulness in patients who may otherwise have had a suboptimal result with medialization alone.

Interestingly, most studies evaluated arytenoid repositioning outcomes measuring results with objective measures alone. In our review of the 22 studies reporting surgical outcomes, only $23 \%$ considered patient-reported outcomes (Fig. 1). This is surprising since the rationale for undergoing medialization and arytenoid adduction is to reverse patient symptoms. Therefore, patient-reported outcomes are critical and the ultimate arbiter of success. In the absence of conclusive data, it is the authors' belief that arytenoid adduction should be considered an adjunct to medialization laryngoplasty when suboptimal voice is achieved with implant placement alone. Such intraoperative adjustment can only be made with, and underscores, the advantage of having an awake patient during the procedure. It would be ideal to be able to determine the need 


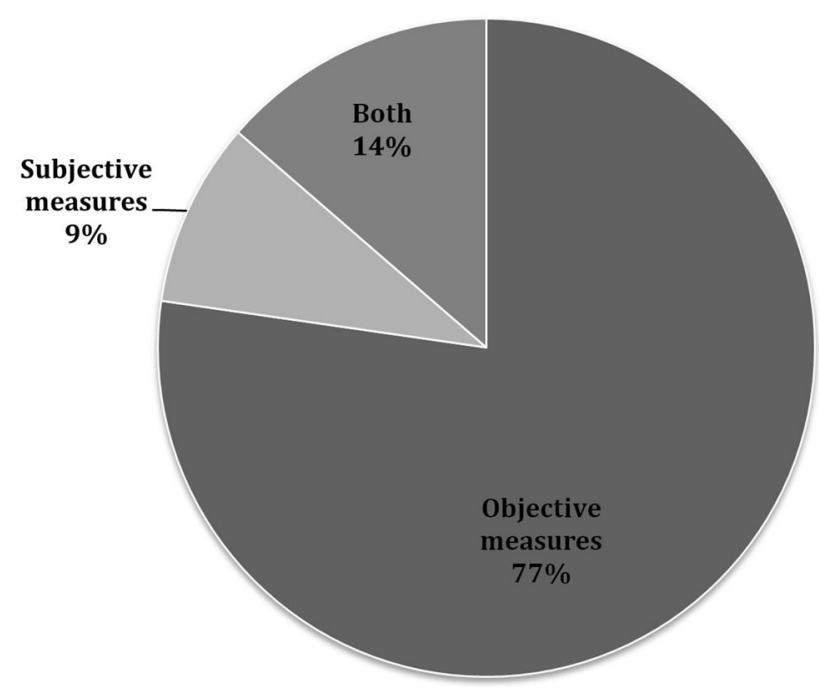

Fig. 1 Proportions of outcome measures in studies of arytenoid repositioning procedures

for arytenoid adduction pre-operatively. An interesting unpublished blinded study was unable to identify any preoperative stroboscopic findings that predicted who ultimately required arytenoid procedures (Garrett, personal communication, 2 January 2014).

\section{Complications}

Overall, the complication rate for type I laryngoplasty in one study was reported to be $14 \%$ and as high as $19 \%$ with the addition of arytenoid adduction, although only 7 and $9 \%$, respectively, required intervention [31]. A national survey of complications by Young et al. [27] reported an overall complication rate of $15.4 \%$, with a revision rate of $6 \%$. The reported implant extrusion rate ranges from 0 to $9.8 \%$ [30, 31, 38, 78, 92]. An increase in complication rates was observed in surgeons who performed fewer then two procedures per year or had an experience of less than ten total cases [38].

Arytenoid procedures carry the highest airway risk because of additional manipulation of the posterior glottis and significantly increased edema [38]. Narajos et al. [97] showed maximal laryngeal edema on the 3rd postoperative day. This is interesting, but most likely not clinically significant because of the rarity of reported airway issues outside of the immediate 24-h postoperative period. Also, in regards to airway management, one must also be careful to identify subtle contralateral motion impairment. Occasionally patients may have a reason for injury to the contralateral side; therefore, videolaryngoscopy should be thoroughly reviewed pre-operatively. This may present with difficulty breathing intraoperatively or new onset stridor with manipulation, but not fully manifest until hours postoperatively. The rate of tracheotomy with arytenoid adduction ranges from 1.7 to $3.5 \%$ with obstruction occurring an average of $9 \mathrm{~h}$ postoperatively [31, 38, 98]. Also, there is one reported allergic reaction to a silicone implant [99]. However, a comparison of silicone, GoreTex ${ }^{\circledR}$, and irradiated cartilage in the rabbit larynx showed the least inflammatory response and best capsule formation with silicone use [100]. Arytenoid adduction is also associated with a risk of intraoperative pyriform sinus perforation [101, 102]. Although commonly cited as a complication of arytenoid adduction, it is rarely reported in the literature $[66,86,88,94]$.

\section{New and Future Directions}

The basic tenets of laryngeal framework surgery have remained constant since its reintroduction in the $1970 \mathrm{~s}$, but techniques, window size and placement, implant materials, and outcomes have dramatically evolved. Nonetheless, many intriguing questions remain. Probably the most controversial topic is the role of arytenoid repositioning. Proper patient selection and development of the most efficient and effective technique still remain largely unanswered because of the limited number of studies looking at patient-centered outcomes. Further studies regarding management of the arytenoid should be performed in a prospective nature considering patient-oriented outcomes in voice, swallowing, and breathing in addition to objective physiologic measures. Patient symptoms drive management of this condition. Thus, patient-level outcomes focused on how the procedure affects symptoms in all domains; laryngeal function and quality of life are the most relevant measures of successful treatment. Moreover, better understanding of the degree of nerve deficit and synkinesis may allow for better pre-operative planning, identify those needing early intervention, and predict which patients will need arytenoid reposition procedures.

Innovations in implant materials and surgical techniques are likely to continue as the laryngeal neuroanatomy and physiology are further clarified. One promising opportunity relates to the availability of cost-effective three-dimensional printers. Theoretically, a computed tomography could recapitulate the exact measurements of the paraglottic space and be used to create customized implants, thereby limiting the amount of intraoperative adjustments needed obtain the best improvement. Finally, the role of reinnervation procedures in framework surgery needs to be further studied. Arytenoid repositioning performed along with reinnervation and temporary injection in the same setting could potentially provide additional benefits [4951]. Restoring tone and bulk to the vocal fold while placing the vocal fold in the optimal position for phonation may provide another option for rehabilitation of the larynx. 


\section{Conclusions}

Laryngeal framework surgery represents the unique intersection of the art and science in laryngology. Significant innovations in implant material and technique continue toward the goal of improving patient outcomes. However, there remain significant surgeon-level variations in how the procedure is performed and very little rigorous comparative effectiveness research that directly compares the efficacy and effectiveness of treatment algorithms and treatments. Progress in the treatment of UVFP will require advances in clinical, basic science, and, importantly, translational research to better understand the initiating neurologic insult, to prognosticate nerve recovery, to analyze potential pro-recovery modulators, and to perform comparative effectiveness research that captures and measures both patient-level and objective measures of voice, swallowing, and breathing improvement.

\section{Compliance with Ethics Guidelines}

Conflict of Interest James J. Daniero, C. Gaelyn Garrett, David O. Francis declare no conflicts of interest

Human and Animal Rights and Informed Consent This article does not contain any studies with human or animal subjects performed by the authors.

\section{References}

Recently published papers that are of particular interest have been highlighted as:

- Of importance

1. Gordon T, Gordon K. Nerve regeneration in the peripheral nervous system versus the central nervous system and the relevance to speech and hearing after nerve injuries. J Commun Disord. 2010;43:274-85.

2. Gacek RR. Localization of laryngeal motor neurons in the kitten. Laryngoscope. 1975;85:1841-61.

3. Wang RC. Three-dimensional analysis of cricoarytenoid joint motion. Laryngoscope. 1998;108:1-17.

4. Rosenthal LH, Benninger MS, Deeb RH. Vocal fold immobility: a longitudinal analysis of etiology over 20 years. Laryngoscope. 2007;117:1864-70.

5. Merati AL, Shemirani N, Smith TL, Toohill RJ. Changing trends in the nature of vocal fold motion impairment. Am J Otolaryngol. 2006;27:106-8.

6. Ko HC, Lee LA, Li HY, Fang TJ. Etiologic features in patients with unilateral vocal fold paralysis in Taiwan. Chang Gung Med J. 2009;32:290-6.

7. Chen HC, Jen YM, Wang CH, Lee JC, Lin YS. Etiology of vocal cord paralysis. ORL J Otorhinolaryngol Relat Spec. 2007;69:167-71.

8. Bhattacharyya N, Fried MP. Assessment of the morbidity and complications of total thyroidectomy. Arch Otolaryngol Head Neck Surg. 2002;128:389-92.

9. Jeannon JP, Orabi AA, Bruch GA, Abdalsalam HA, Simo R. Diagnosis of recurrent laryngeal nerve palsy after thyroidectomy: a systematic review. Int J Clin Pract. 2009;63:624-9.
10. Woo P, Pearl AW, Hsiung MW, Som P. Failed medialization laryngoplasty: management by revision surgery. Otolaryngol Head Neck Surg. 2001;124(6):615-21.

11. Kriskovich MD, Apfelbaum RI, Haller JR. Vocal fold paralysis after anterior cervical spine surgery: incidence, mechanism, and prevention of injury. Laryngoscope. 2000;110:1467-73.

12. Jung A, Schramm J, Lehnerdt K, Herberhold C. Recurrent laryngeal nerve palsy during anterior cervical spine surgery: a prospective study. J Neurosurg Spine. 2005;2:123-7.

13. Davies L, Welch HG. Increasing incidence of thyroid cancer in the United States, 1973-2002. JAMA. 2006;295:2164-7.

14. Marawar S, Girardi FP, Sama AA, et al. National trends in anterior cervical fusion procedures. Spine. 2010;35:1454-9.

15. SEER Cancer Statistics Review 19752007.

16. - Cohen SM, Kim J, Roy N, Asche C, Courey M. The impact of laryngeal disorders on work-related dysfunction. Laryngoscope 2012; 122:1589-94. Evaluates the economic impact of laryngeal disorders including unilateral vocal fold paralysis. Provides a quantitative analysis of short-term disability payments and days lost from work.

17. Cohen SM, Dupont WD, Courey MS. Quality-of-life impact of non-neoplastic voice disorders: a meta-analysis. Ann Otol Rhinol Laryngol. 2006;115:128-34.

18. Fang TJ, Li HY, Gliklich RE, Chen YH, Wang PC, Chuang HF. Quality of life measures and predictors for adults with unilateral vocal cord paralysis. Laryngoscope. 2008;118:1837-41.

19. Brunner E, Friedrich G, Kiesler K, Chibidziura-Priesching J, Gugatschka M. Subjective breathing impairment in unilateral vocal fold paralysis. Folia Phoniatr Logop. 2011;63:142-6.

20. Bhattacharyya N, Kotz T, Shapiro J. Dysphagia and aspiration with unilateral vocal cord immobility: incidence, characterization, and response to surgical treatment. Ann Otol Rhinol Laryngol. 2002;111:672-9.

21. Sulica L. The natural history of idiopathic unilateral vocal fold paralysis: evidence and problems. Laryngoscope. 2008;118: 1303-7.

22. - Rickert SM, Childs LF, Carey BT, Murry T, Sulica L. Laryngeal electromyography for prognosis of vocal fold palsy: a meta-analysis. Laryngoscope 2012; 122:158-61. Review examining the ability of laryngeal electromyography (LEMG) to predict recovery in vocal fold paralysis. It showed that LEMG is not useful in predicting recovery, but identifies patients with severe injury that will not recover function. This supports early definitive intervention in patients with high-grade injury on LEMG.

23. Paniello RC, Edgar JD, Kallogjeri D, Piccirillo JF. Medialization versus reinnervation for unilateral vocal fold paralysis: a multicenter randomized clinical trial. Laryngoscope 2011; 121:2172-79. Small randomized trial comparing reinnervation and medialization laryngoplasty. It showed similar voice outcomes in the two groups at 1-year follow-up. Better reinnervation results were demonstrated in patients under 52 years old.

24. Spector BC, Netterville JL, Billante C, Clary J, Reinisch L, Smith TL. Quality-of-life assessment in patients with unilateral vocal cord paralysis. Otolaryngol Head Neck Surg. 2001;125:176-82.

25. Payr E. Plastik am schildknorpel zur Behebung der Folgen einseitiger Stimmbandlahmung. Dtsch Med Wochenschr. 1915;43: 1265-70.

26. Isshiki N, Morita $\mathrm{H}$, Okamura $\mathrm{H}$, Hiramoto M. Thyroplasty as a new phonosurgical technique. Acta Otolaryngol. 1974;78:451-7.

27. Young VN, Zullo TG, Rosen CA. Analysis of laryngeal framework surgery: 10-year follow-up to a national survey. Laryngoscope. 2010;120:1602-8.

28. Isshiki N, Tanabe M, Sawada M. Arytenoid adduction for unilateral vocal cord paralysis. Archiv Otolaryngol. 1978;104: $555-8$. 
29. Koufman JA. Laryngoplasty for vocal cord medialization: an alternative to Teflon. Laryngoscope. 1986;96:726-31.

30. Netterville JL, Stone RE, Luken ES, Civantos FJ, Ossoff RH. Silastic medialization and arytenoid adduction: the Vanderbilt experience. A review of 116 phonosurgical procedures. Ann Otol Rhinol Laryngol. 1993;102:413-24.

31. Abraham MT, Gonen M, Kraus DH. Complications of type I thyroplasty and arytenoid adduction. Laryngoscope. 2001;111: 1322-9.

32. Sodersten M, Lindestad PA. A comparison of vocal fold closure in rigid telescopic and flexible fiberoptic laryngostroboscopy. Acta Otolaryngol. 1992;112:144-50.

33. Fleischer S, Schade G, Hess MM. Office-based laryngoscopic observations of recurrent laryngeal nerve paresis and paralysis. Ann Otol Rhinol Laryngol. 2005;114:488-93.

34. Gray SD, Barkmeier J, Jones D, Titze I, Druker D. Vocal evaluation of thyroplastic surgery in the treatment of unilateral vocal fold paralysis. Laryngoscope. 1992;102:415-21.

35. Graboyes EM, Bradley JP, Meyers BF, Nussenbaum B. Efficacy and safety of acute injection laryngoplasty for vocal cord paralysis following thoracic surgery. Laryngoscope. 2011;121:2406-10.

36. Hendricker RM, de Silva BW, Forrest LA. Gore-Tex medialization laryngoplasty for treatment of dysphagia. Otolaryngol Head Neck Surg. 2010;142:536-9.

37. Nayak VK, Bhattacharyya N, Kotz T, Shapiro J. Patterns of swallowing failure following medialization in unilateral vocal fold immobility. Laryngoscope. 2002;112:1840-4.

38. Rosen CA. Complications of phonosurgery: results of a national survey. Laryngoscope. 1998;108:1697-703.

39. Paniello RC, Dahm JD. Reversibility of medialization laryngoplasty. An experimental study. Ann Otol Rhinol Laryngol. 1997; 106:902-8.

40. Saarinen A, Sovijarvi AR, Karhumaki L, Rihkanen H. Medialization of paralyzed vocal fold does not increase respiratory resistance measured by impulse oscillometry. Eur Arch Otorhinolaryngol. 2007;264:1323-7.

41. D’Alatri L, Galla S, Rigante M, Antonelli O, Buldrini S, Marchese MR. Role of early voice therapy in patients affected by unilateral vocal fold paralysis. J Laryngol Otol. 2008;122:936-41.

42. Miller S. Voice therapy for vocal fold paralysis. Otolaryngol Clin North Am. 2004;37:105-19.

43. Schindler A, Bottero A, Capaccio P, Ginocchio D, Adorni F, Ottaviani F. Vocal improvement after voice therapy in unilateral vocal fold paralysis. J Voice. 2008;22:113-8.

44. Heuer RJ, Sataloff RT, Emerich K, et al. Unilateral recurrent laryngeal nerve paralysis: the importance of "pre-operative" voice therapy. J Voice. 1997;11:88-94.

45. Sulaiman OA, Gordon T. Effects of short- and long-term Schwann cell denervation on peripheral nerve regeneration, myelination, and size. Glia. 2000;32:234-46.

46. Burnett MG, Zager EL. Pathophysiology of peripheral nerve injury: a brief review. Neurosurg Focus. 2004;16:E1.

47. Fugleholm K, Schmalbruch H, Krarup C. Early peripheral nerve regeneration after crushing, sectioning, and freeze studied by implanted electrodes in the cat. J Neurosci. 1994;14:2659-73.

48. Seddon HJ, Medawar PB, Smith H. Rate of regeneration of peripheral nerves in man. J Physiol. 1943;102:191-215.

49. Prades JM, Dubois MD, Dumollard JM, et al. Morphological and functional asymmetry of the human recurrent laryngeal nerve. Surg Radiol Anat. 2012;34:903-8.

50. Tsunoda K, Kikkawa YS, Kumada M, Higo R, Tayama N. Hoarseness caused by unilateral vocal fold paralysis: how long should one delay phonosurgery? Acta Otolaryngol. 2003;123: $555-6$.

51. Williams RG. Idiopathic recurrent laryngeal nerve paralysis. J Laryngol Otol. 1959;73:161-6.
52. Abraham MT, Bains MS, Downey RJ, Korst RJ, Kraus DH. Type I thyroplasty for acute unilateral vocal fold paralysis following intrathoracic surgery. Ann Otol Rhinol Laryngol. 2002;111:667-71.

53. Pellegrino C, Franzini C. An electron microscope study of denervation atrophy in red and white skeletal muscle fibers. J Cell Biol. 1963;17:327-49.

54. Hnik P. Rate of denervation muscle atrophy. In: Gutmann E, editor. Rate of denervation muscle atrophy. Berlin: Springer; 1962.

55. Sittel C, Stennert E, Thumfart WF, Dapunt U, Eckel HE. Prognostic value of laryngeal electromyography in vocal fold paralysis. Arch Otolaryngol Head Neck Surg. 2001;127:155-60.

56. Munin MC, Rosen CA, Zullo T. Utility of laryngeal electromyography in predicting recovery after vocal fold paralysis. Arch Phys Med Rehabil. 2003;84:1150-3.

57. Parnes SM, Satya-Murti S. Predictive value of laryngeal electromyography in patients with vocal cord paralysis of neurogenic origin. Laryngoscope. 1985;95:1323-6.

58. Grosheva M, Wittekindt C, Pototschnig C, Lindenthaler W, Guntinas-Lichius O. Evaluation of peripheral vocal cord paralysis by electromyography. Laryngoscope. 2008;118:987-90.

59. Hydman J, Bjorck G, Persson JK, Zedenius J, Mattsson P. Diagnosis and prognosis of iatrogenic injury of the recurrent laryngeal nerve. Ann Otol Rhinol Laryngol. 2009;118:506-11.

60. Gupta SR, Bastian RW. Use of laryngeal electromyography in prediction of recovery after vocal cord paralysis. Muscle Nerve. 1993;16:977-8.

61. Elez F, Celik M. The value of laryngeal electromyography in vocal cord paralysis. Muscle Nerve. 1998;21:552-3.

62. Min YB, Finnegan EM, Hoffman HT, Luschei ES, McCulloch TM. A preliminary study of the prognostic role of electromyography in laryngeal paralysis. Otolaryngol Head Neck Surg. 1994;111:770-5.

63. Leder SB, Ross DA. Incidence of vocal fold immobility in patients with dysphagia. Dysphagia. 2005;20:163-7.

64. Leder SB, Suiter DM, Duffey D, Judson BL. Vocal fold immobility and aspiration status: a direct replication study. Dysphagia. 2012;27:265-70.

65. Jang YY, Lee SJ, Jeon JY, Lee SJ. Analysis of video fluoroscopic swallowing study in patients with vocal cord paralysis. Dysphagia. 2012;27:185-90.

66. Carrau RL, Pou A, Eibling DE, Murry T, Ferguson BJ. Laryngeal framework surgery for the management of aspiration. Head Neck. 1999;21:139-45.

67. Bhattacharyya N, Kotz T, Shapiro J. The effect of bolus consistency on dysphagia in unilateral vocal cord paralysis. Otolaryngol Head Neck Surg. 2003;129:632-6.

68. Jalisi S, Netterville JL. Rehabilitation after cranial base surgery. Otolaryngol Clin North Am. 2009;42:49-56.

69. Schwaber MK, Netterville JL, Coniglio JU. Complications of skull base surgery. Ear Nose Throat J. 1991;70(648-654): 659-60.

70. Netterville JL, Fortune S, Stanziale S, Billante CR. Palatal adhesion: the treatment of unilateral palatal paralysis after high vagus nerve injury. Head Neck. 2002;24:721-30.

71. Pou AM, Carrau RL, Eibling DE, Murry T. Laryngeal framework surgery for the management of aspiration in high vagal lesions. Am J Otolaryngol. 1998;19:1-7.

72. Ollivere B, Duce K, Rowlands G, Harrison P, O'Reilly BJ. Swallowing dysfunction in patients with unilateral vocal fold paralysis: aetiology and outcomes. J Laryngol Otol. 2006;120: 38-41.

73. Cavusoglu H, Tuncer $\mathrm{C}$, Tanik $\mathrm{C}$, et al. The impact of automatic retractors on the esophagus during anterior cervical surgery: an experimental in vivo study in a sheep model. J Neurosurg Spine. 2009;11:547-54. 
74. Heese O, Fritzsche E, Heiland M, Westphal M, Papavero L. Intraoperative measurement of pharynx/esophagus retraction during anterior cervical surgery. Part II: perfusion. Euro Spine J. 2006;15:1839-43.

75. Winslow CP, Winslow TJ, Wax MK. Dysphonia and dysphagia following the anterior approach to the cervical spine. Arch Otolaryngol Head Neck Surg. 2001;127:51-5.

76. Wanamaker JRN, Netterville JL, Ossoff RH. Phonosurgery: silastic medialization for unilateral vocal fold paralysis. Oper Tech Otolaryngol Head Neck Surg. 1993;4:207-17.

77. McCulloch TM, Hoffman HT. Medialization laryngoplasty with expanded polytetrafluoroethylene. Surgical technique and preliminary results. Ann Otol Rhinol Laryngol. 1998;107:427-32.

78. Cotter CS, Avidano MA, Crary MA, Cassisi NJ, Gorham MM. Laryngeal complications after type 1 thyroplasty. Otolaryngol Head Neck Surg. 1995;113:671-3.

79. Isshiki N. Vocal mechanics as the basis for phonosurgery. Laryngoscope. 1998;108:1761-6.

80. Woodson G. Evolving concepts of laryngeal paralysis. J Laryngol Otol. 2008;122:437-41.

81. Hoffman MR, Devine EE, McCulloch TM, Jiang JJ. Excised larynx evaluation of wedge-shaped adjustable balloon implant for minimally invasive type I thyroplasty. Laryngoscope. 2013. doi:10.1002/lary.24409.

82. Hoffman MR, Witt RE, McCulloch TM, Jiang JJ. Preliminary investigation of adjustable balloon implant for type I thyroplasty. Laryngoscope. 2011;121:793-800.

83. Isshiki N. Progress in laryngeal framework surgery. Acta Otolaryngol. 2000;120:120-7.

84. - Hoffman MR, Surender K, Chapin WJ, Witt RE, McCulloch TM, Jiang JJ. Optimal arytenoid adduction based on quantitative real-time voice analysis. Laryngoscope 2011; 121:339-45. Study evaluating varying degrees of arytenoid adduction in excised canine larynges. Demonstrated that the optimal angle of rotation can be determined by real-time acoustic and aerodynamic parameters.

85. Miller FRB, Bryant GL, Netterville JL. Arytenoid adduction in vocal fold paralysis. Oper Tech Otolaryngol Head Neck Surg. 1999;10:36-41.

86. Mitchell JR, McRae BR, Halum SL. Localization of the muscular process for arytenoid adduction surgery. Laryngoscope. 2009;119:631-4.

87. Armin BB, Head C, Berke GS, Chhetri DK. Useful landmarks in arytenoid adduction and laryngeal reinnervation surgery. Laryngoscope. 2006;116:1755-9.

88. Zeitels SM, Hochman I, Hillman RE. Adduction arytenopexy: a new procedure for paralytic dysphonia with implications for implant medialization. Ann Otol Rhinol Laryngol Suppl. 1998;173:2-24.
89. Zeitels SM, Hillman RE, Desloge RB, Bunting GA. Cricothyroid subluxation: a new innovation for enhancing the voice with laryngoplastic phonosurgery. Ann Otol Rhinol Laryngol. 1999;108:1126-31.

90. McNamar J, Montequin DW, Welham NV, Dailey SH. Aerodynamic, acoustic, and vibratory comparison of arytenoid adduction and adduction arytenopexy. Laryngoscope. 2008;118:552-8.

91. Chester MW, Stewart MG. Arytenoid adduction combined with medialization thyroplasty: an evidence-based review. Otolaryngol Head Neck Surg. 2003;129:305-10.

92. Kraus DH, Orlikoff RF, Rizk SS, Rosenberg DB. Arytenoid adduction as an adjunct to type I thyroplasty for unilateral vocal cord paralysis. Head Neck. 1999;21:52-9.

93. - Li AJ, Johns MM, Jackson-Menaldi C, et al. Glottic closure patterns: type I thyroplasty versus type I thyroplasty with arytenoid adduction. J Voice 2011; 25:259-64. Study of blinded reviewers evaluating glottic closure patterns in patients who underwent thyroplasty with or without arytenoid adduction. preoperative closure patterns predicted the necessity of arytenoid adduction to improve voice quality; however, no postoperative differences on laryngoscopy were noted with arytenoid adduction.

94. Murata T, Yasuoka Y, Shimada T, et al. A new and less invasive procedure for arytenoid adduction surgery: endoscopic-assisted arytenoid adduction surgery. Laryngoscope. 2011;121:1274-80.

95. Hoffman MR, Witt RE, Chapin WJ, McCulloch TM, Jiang JJ. Multiparameter comparison of injection laryngoplasty, medialization laryngoplasty, and arytenoid adduction in an excised larynx model. Laryngoscope. 2010;120:769-76.

96. Mortensen M, Carroll L, Woo P. Arytenoid adduction with medialization laryngoplasty versus injection or medialization laryngoplasty: the role of the arytenoidopexy. Laryngoscope. 2009;119:827-31.

97. Narajos N, Toya Y, Kumai Y, Sanuki T, Yumoto E. Videolaryngoscopic assessment of laryngeal edema after arytenoid adduction. Laryngoscope. 2012;122:1104-8.

98. Weinman EC, Maragos NE. Airway compromise in thyroplasty surgery. Laryngoscope. 2000;110:1082-5.

99. Hunsaker DH, Martin PJ. Allergic reaction to solid silicone implant in medial thyroplasty. Otolaryngol Head Neck Surg. 1995;113:782-4.

100. Ustundag E, Boyaci Z, Keskin G, Kaur A, Ozkarakas H. Soft tissue response of the larynx to silicone, Gore-Tex, and irradiated cartilage implants. Laryngoscope. 2005;115:1009-14.

101. McCulloch TM, Hoffman HT, Andrews BT, Karnell MP. Arytenoid adduction combined with Gore-Tex medialization thyroplasty. Laryngoscope. 2000;110:1306-11.

102. Slavit DH, Maragos NE. Physiologic assessment of arytenoid adduction. Ann Otol Rhinol Laryngol. 1992;101:321-7. 